\title{
Residual life assessment of welded joints in the girth weld root
}

\author{
Leonid Shron ${ }^{1, *}$, Vladimir Bogutsky ${ }^{1}$, Elmar Yagyaev ${ }^{2}$, and Illa Tabolin ${ }^{3}$ \\ ${ }^{1}$ Sevastopol State University, 299053, str. University, 33, Sevastopol, Russia \\ ${ }^{2}$ Crimean Engineering and Pedagogical University named after Fevzi Yakubov, per.Uchebnyj, 8, \\ Simferopol, 295015, Russia \\ ${ }^{3}$ Closed joint-stock company «Chelyabinsk interactive cable networks", st. Ordzhonikidze, 54-b, \\ Chelyabinsk, Russia
}

\begin{abstract}
The article analyzes the influence of the structural crack-like defect in the girth weld root welding the bottom to the collector body on the crack growth resistance. A mathematical model has been developed to estimate the residual life resource of welded joints with an internal crack-like defect in the root of the weld under creep. Results of metallographic studies of bench tests results for the welded joints long-term strength formed the basis of the calculation model. In all tested models the cracks start at the top of the narrow structural gap in the weld root and develop along almost the entire weld axis, perpendicular to the wall of the pipe and have a discrete growth pattern. As a result, discrete rupture of material occurs on a length of rc, and the initial crack is incremented by this length. After that, a "plastic core" of rc length is formed in the newly formed crack tip, additional damage is accumulated, and the crack development process continues until the stress level in the section weakened by the crack reaches a critical value. The obtained dependence characterizes the crack growth time from the initial crack-like defect to its critical length.
\end{abstract}

\section{Introduction}

The experience of operating steam boilers indicates cases of sudden detachment of the bottom from the end of the collector along the weld metal, on the inside of which there was a linear crack-like defect in the form of lack of fusion in the weld's root. This is explained by the fact that the bottom wall, loaded with internal pressure, imitates the conditions of tight sealing and leads to the appearance of a concentrated bending moment that additionally affects the value of axial tensile stresses near the tip of the crack-like defect in the root of the weld. This problem is a pressing challenge and requires a solution.

Numerous experimental and theoretical studies are devoted to the study of crack growth under creep conditions [1-6]. Obviously, a reliable interpretation of the experimental data is impossible without determining the stress condition not only near the crack tip, but also on the path of its propagation. In this work, when determining the stress condition in the vicinity of the tip of a stationary crack loaded under steady creep conditions, we used the approach described in [7]. According to the works [7] and [8], we assume that the stress

\footnotetext{
* Corresponding author: shronlb@mail.ru
} 
diagram $\sigma_{y}$ on the extension of the crack consists of three areas: nonlinear deformations and the implementation of the fracture mechanism ( $r_{c}$ length), singular $\left(r_{s}\right.$ length), regular $\left(b-l-r_{s}\right.$ length) and has the nature of the dependence presented in Fig 1.

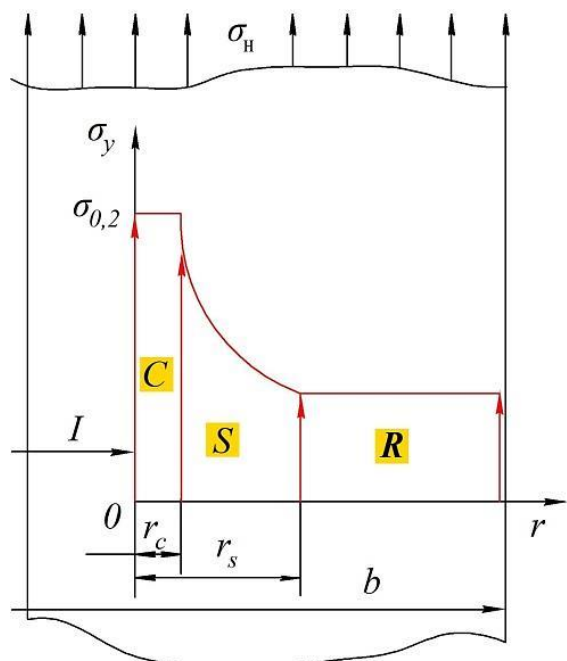

Fig. 1. Distribution of $\sigma_{y}$ stress along the plane of the normal fracture crack: $\mathrm{C}$ is an area of nonlinear deformations, $\mathrm{S}$ is an area of singular stresses, $\mathrm{R}$ is an area of regular stresses.

On the $\left[0 r_{c}\right]$ segment, the stress $\sigma_{y}=\sigma_{0,2}^{c}$, where $\sigma_{0,2}^{c}$ is the yield strength of the material at creep temperature. Such a redistribution of the asymptotic $\sigma_{y}$ stresses on the small-scale yield segment is the well-known Irwin approximation [9] with the only difference that in our case the $r_{c}$ and $r_{s}$ segments are subject to determination, as well as the $f_{I}(\lambda)$ correction function. Here, the relative (dimensionless) crack length is still represented as $\lambda=l_{/ b}$.

\section{Materials and Methods}

According to the theory of plasticity. power law hardening elastoplastic bodies have the components of the stress tensor near the tip of a stationary crack determined by the Hutchinson-Rice-Rosengren singular solution (the so-called HRR asymptotics, $[10,11]$ ). For steady creep (for $\mathrm{n}>1$ ), the valid asymptotic behavior is:

$$
\sigma_{i j} \sim r^{-\frac{1}{n+1}}
$$

Preserving the stress dimension in (1) and assuming the structure of the stress intensity factor (SIF) in the form:

$$
K_{I}=\sigma_{\mathrm{H}} \cdot l^{\frac{1}{n+1}} \cdot f_{I}(\lambda),
$$

we write the approximate expression for the $\sigma_{y}$ stress at the crack extension in the form:

$$
\sigma_{y} \approx \sigma_{\mathrm{H}} \cdot\left(\frac{r}{l}\right)^{-\frac{1}{n+1}} \cdot f_{I}(\lambda) \text {. }
$$


Multiplying and dividing expression (2) by $(2 \pi)^{\frac{1}{n+1}}$, assuming $n=1$, we obtain, up to a constant factor $\sqrt{2}$, the well-known expression of $\sigma_{y}$ for a normal fracture crack in linear fracture mechanics:

$$
\sigma_{y}=\frac{K_{I}}{\sqrt{2 \pi \cdot r}} \cdot \varphi_{y}(\theta)
$$

where $K_{I}=\sigma_{\mathrm{H}} \sqrt{\pi \cdot l} \cdot f_{I}(\lambda), \varphi_{y}(\theta)$ is a limited function of the polar angle, assuming a value of 1 at $\theta=0^{\circ}$. Of course, the formal approach to constructing asymptotic expression (2) demonstrated above is a rough approximation, but at the same time, for engineering applications, it not only qualitatively reflects the stress distribution $\sigma_{y}$ over the entire crack cross section propagation, but also allows one to estimate the residual life of specific structural elements operating under creep conditions .

Using the approach developed in [7], as applied to the problem posed, a nonlinear algebraic equation is obtained for the desired quantity $r_{s}$ with three variable parameters $\lambda$, $\mu$, $n$ in the form:

$$
Y=Y\left(r_{s}, \lambda, \mu, n\right)
$$

$\mu=\sigma_{\mathrm{H}} / \sigma_{0,2}^{c}, \sigma_{\mathrm{H}}$ is nominal tensile stress in the undamaged section of the pipe wall (Fig. 1). The numerical solution of (4) with respect to $r_{s}$ allows us to find $r_{c}$ and $F_{I}(\lambda)$ by the following formulas:

$$
\begin{gathered}
r_{c}(\lambda)=r_{s}(\lambda) \cdot\left[\frac{\mu \cdot(1-\lambda)}{1-\lambda-\lambda \cdot r_{s}(\lambda)}\right]^{1+n}, \\
F_{I}(\lambda)=\left[\frac{1-\lambda}{1-\lambda-\lambda \cdot r_{s}(\lambda)}\right] \cdot\left[2 \cdot r_{s}(\lambda)\right]^{N}, \\
N=\frac{1}{1+n} .
\end{gathered}
$$

Figure 2a shows the results of the calculation of the correction function $F_{I}(\lambda)$ in a graph. The calculation uses the data obtained during bench tests for the long-term strength of the welded joint of the bottom and pipe wall in Fig $2 \mathrm{~b}$. The initial relative length of the cracklike defect is $\lambda=0.315$; the parameters $n$ and $\mu$ are 2 and 0.051 , respectively.

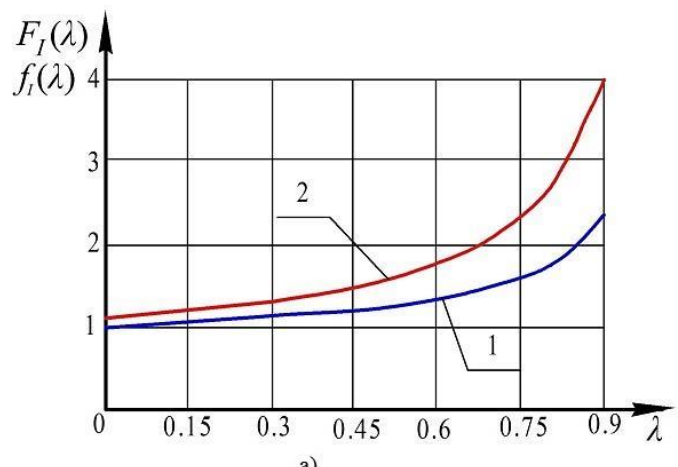

a)

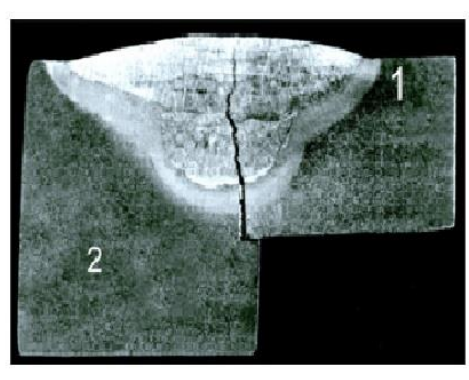

b)

Fig. 2. a) Graphs of correction functions: curve $1-f_{l}(\lambda)$, linear asymptotics, $n=1, \mu=0$, curve $2-F_{I}(\lambda)$, non-linear asymptotics, $n=2, \mu=0.0513$; b) The weld template of the pipe joint (2) with the bottom (1) (model material is $12 \mathrm{X} 1 \mathrm{MF}$ steel, temperature is $600 \mathrm{C}$, test time is 3000 hours, increase by 100 ). 
We verify the validity of the obtained expressions (4), (5) and (6) with the boundary value $\lambda=0$. In this case, the external contour (configuration) of the loaded element does not affect the intensity of the stresses near the crack tip (the so-called case of a body of infinite size with a normal fracture crack).

When $\lambda=0$, expression (4) admits a transformation to the form:

$$
r_{\mathrm{s}}(\mu, n)=\frac{n}{1+n-\mu^{n}}
$$

and, as a consequence of this,

$$
F_{I}(\mu, n)=\left[2 \cdot r_{s}(\mu, n)\right]^{N}
$$

From (7) and (8) it is easy to see that the value of the correction function is $F_{I}(\mu=0$, $n=1)=1$ and coincides with the value $f_{I}(\lambda=0)=1$ in the framework of linear fracture mechanics, which is quite acceptable for engineering applications taking into account the assumptions made above.

Figure 3 shows the family of curves $F_{I}(\mu, n)$ for various discrete ratios of $\mu$ and $n$ parameters.

For engineering applications, it can be assumed that curves 1 and 4 limit the range of values $F_{I}(\mu, n)$ for acceptable ratios of the parameters of $\mu$ and $n$. We also note that as the parameter $n$ increases, the family of curves $F_{I}(\mu, n)$ asymptotically approaches the lower curve $(\mu=0)$.

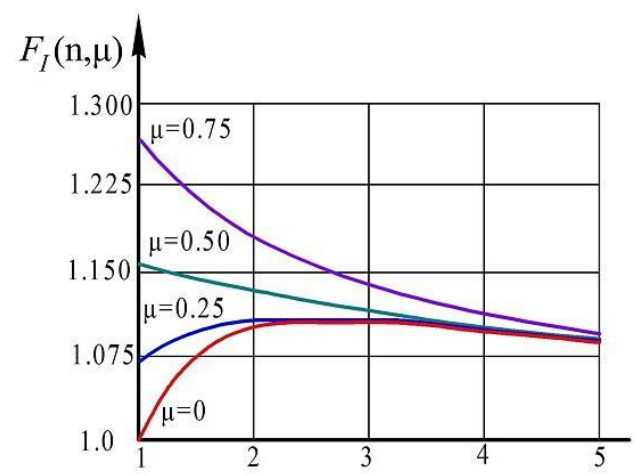

Fig. 3. Family of correction functions $F_{I}(\mu, n)$ at $\lambda=0$.

\section{Result and Discussion}

The results obtained allow us to proceed to the development of an engineering method for estimating the resource of welded joints of the bottoms of boiler collectors with a crack-like defect in the root of the girth weld.

A significant number of works $[12,13]$ are devoted to the construction of calculated phenomenological models of fracture of materials under creep conditions. The main task of such calculation models is to determine the time to failure of a loaded structural element. The calculation models are focused on the description of brittle fracture during creep proceeding by nucleation of pores along the grain boundaries orthogonal to the direction of maximum tensile stresses $\sigma_{\max }$, with subsequent formation of pore chains, their merging first into separate microcracks, and then combining into one macrocrack, the development of which leads to complete destruction of the structural element. In the work [12], when describing the fracture mechanism, a scalar parameter $\omega$ was introduced; it characterizes the damage to the material and obeys a kinetic equation of the form: 


$$
\frac{d \omega}{d t}=A \cdot\left[\frac{\sigma_{\max }}{1-\omega}\right]^{n},
$$

where $A$ and $n$ are material constants, determined from the long-term strength diagram in the coordinates " $\operatorname{Lg}(\sigma) \sim \operatorname{Lg}(t)$ "; the range of variation of the parameter $\omega$ lies in the interval $0 \leq \omega<1$.

Equation (9) is easily integrated (the parameter $\omega$ depends only on time $t$ ), which allows us to obtain an expression for finding the time to destruction $t_{c}$ depending on the level of maximum tensile stresses $\sigma_{\max }$ :

$$
t_{c}=\left[A \cdot(1+n) \cdot \sigma_{\max }\right]^{-1} .
$$

It should be noted that subsequently several modifications of the kinetic equation (9) were proposed, with few exceptions, they concerned the modification of the right-hand side by combination with the parameter $\omega$ and the introduction of additional constants of material, without affecting the basic structure of the equation. This circumstance confirms the high efficiency of the structure of the kinetic equation (9). A fairly complete review of modern scientific literature on the issues of long-term destruction is given in [14-20].

The modification of equation (9) is based on the following considerations. We use the concept of the scalar damage function $\psi$, fully preserving the structure of the kinetic equation (9):

$$
\frac{d \psi}{d t}=A\left[\frac{\sigma_{\max }}{1-\psi}\right]^{n}
$$

We assume that the scalar function $\psi$ is directly proportional to the modulus of a radius vector $\vec{r}$, that is

$$
\psi=\sqrt{\vec{r} \cdot \vec{r}} .
$$

and write the full differential of the expression (12):

$$
d \psi=\frac{\vec{r} \cdot d \vec{r}}{\sqrt{\vec{r} \cdot \vec{r}}}
$$

There is the sign of scalar multiplication between vectors $\vec{r}$ in the last two expressions. Next, we represent the radius vector in the form of a linear combination of vectors with components $\omega$ and $\lambda$ :

$$
\vec{r}=\omega \cdot \vec{i}+\lambda \cdot \vec{j}
$$

where $\vec{i}$ and $\vec{j}$ are unit vectors. Then (14) gives the expressions for $d \vec{r}$ :

and scalar product of vectors $\vec{r} \cdot \vec{r}$ :

$$
d \vec{r}=\vec{i} \cdot d \omega+\vec{j} \cdot d \lambda,
$$

$$
\vec{r} \cdot \vec{r}=\omega^{2}+\lambda^{2}
$$

In view of (15) and (16), we rewrite expression (13):

$$
d \psi=\frac{\omega \cdot d \omega+\lambda \cdot d \lambda}{\sqrt{\omega^{2}+\lambda^{2}}}
$$

Dividing both sides of equality (17) by $d t$, we obtain in final form a modified expression for the kinetic damage equation: 


$$
\omega \cdot \frac{d \omega}{d t}+\lambda \cdot \frac{d \lambda}{d t}=\frac{A \cdot \sigma_{\max }^{n} \sqrt{\omega^{2}+\lambda^{2}}}{\left[1-\sqrt{\omega^{2}+\lambda^{2}}\right]^{n}}
$$

As a result of the proposed modification, the structure of the kinetic damage equation became "two-speed" (two-parameter), where $d \omega / d t$ is the growth rate of $\omega$ and $d \lambda / d t$ is the growth rate of the relative crack length $\lambda$. It is easy to see that at $\lambda=0$, equation (18) goes over into the original evolutionary damage equation (9). At $\omega=0$, equation (18) is transformed into an expression that can be interpreted as the kinetic equation of damage during crack growth under conditions of high-temperature corrosion.

To describe the fracture mechanism based on equation (18), we make the necessary explanations regarding the domain of definition of the function $\psi$. As follows from equation (18), the modulus of the scalar function $\psi$, like the parameters $\omega$ and $\lambda$, takes values from the range $0 \leq|\psi|<1$. It follows that the limiting damage curve $\psi$ is described by the equation of the circle and is located in the first quarter of the plane coordinate system $\lambda 0 \omega$. Figure 4 illustrates this conclusion.

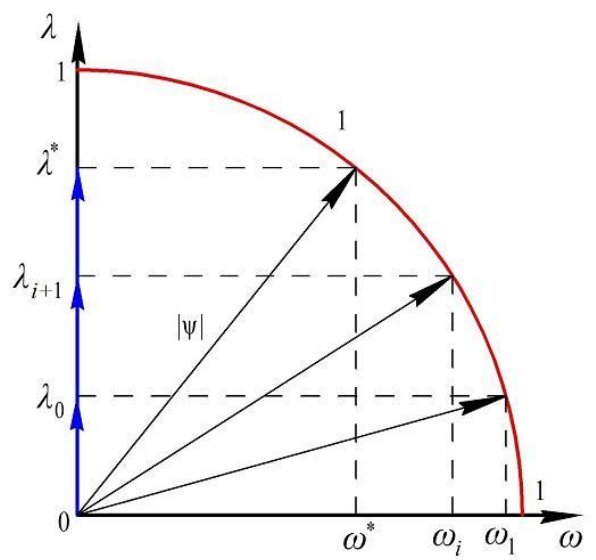

Fig. 4. Trace of the fracture curve 1 in the coordinate plane $\omega 0 \lambda$.

The destruction criterion takes the form:

$$
|\psi|=1
$$

We proceed to formulate the fracture mechanism based on equation (18). Analysis of service fractures and the results of bench tests for the long-term strength of full-scale models indicate a discrete nature of the growth of a creep crack. In all tested models, cracks originate from the top of the narrow structural gap at the root of the weld and develop almost along the axis of the weld, perpendicular to the pipe wall. All cracks are intergranular in nature. Figure $5 \mathrm{a}, 5 \mathrm{~b}$ and $5 \mathrm{c}$ show the results of metallographic studies of a particular model, prematurely withdrawn from testing after 5762 hours. 


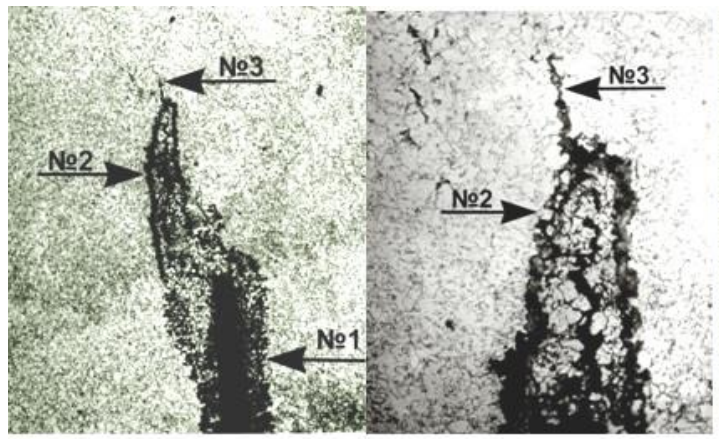

a) b)

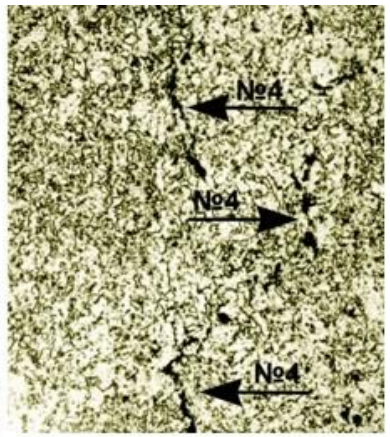

c)

Fig. 5. a) the structure of the weld metal near the initial defect No. 1, the growth of yield crack No. 2, the formation of microcracks No. 3 (st.12X1MF, temperature $699{ }^{\circ} \mathrm{C}$, test time 5762 hours, x100); b) the structure of the weld metal near the creep crack No. 2, the formation of microcracks No. 3 (x500); c) the structure of the weld metal near the crack, the pore fusion in a microcrack No. 4 (x500).

Figure 5a illustrates the evolution of the growth of a creep crack: from the crack to which arrow No. 1 is assigned, and then to cracks No. 2 and No. 3. Such a description of the process at the crack tip with steady creep shows that at the stage of a continuous increase in the parameter $\omega$ from $\omega_{i}$ (current value $\left|\psi_{I}\right|<1$ ) to $\omega_{i+1}$, at which $\left|\psi_{I+1}\right|=1$, the growth rate of relative length crack equals to zero $(d \lambda / d t=0)$. This circumstance makes it possible to explicitly integrate the modified kinetic equation (18) and to obtain an expression for finding the time interval between the current increments of the relative crack length. As a result of integration, we get:

$$
\begin{aligned}
& t_{i+1}-t_{i}=\frac{\left[1-\sqrt{\omega_{i}^{2}+\lambda_{i}^{2}}\right]^{1+n}}{(1+n) \cdot A \cdot \sigma_{\max }^{n}}- \\
& -\frac{\left[1-\sqrt{\omega_{i+1}^{2}+\lambda_{i+1}^{2}}\right]^{1+n}}{(1+n) \cdot A \cdot \sigma_{\max }^{n}}
\end{aligned}
$$

To estimate the residual life using expression (20), we assume the nature of the distribution of $\omega$ along the crack propagation plane.

First, note that in smooth bodies with a constant cross-sectional area, tensile stresses $\sigma_{y}$ are uniformly distributed in the section plane orthogonal to the applied force and maximum stresses $\sigma_{\max }=\sigma_{y}$.

\section{Conclusion}

The process of pore nucleation and coalescence, as well as the value of the parameter $\omega$ at each fixed time $t_{i}$, can also be taken uniformly distributed along the cross section of the part, which, in turn, leads to a similarity of the stress diagrams $\sigma_{\max }$ and parameter $\omega$ as plane figures.

Following these assumptions, we assume that the parameter $\omega$ varies along the horizontal axis or (see Fig. 1) in the form of a plane figure similar to the stress diagram $\sigma_{y}$, i.e. $\omega(r) \sim \sigma_{y}(r)$. On the $\left[o r_{c}\right]$ segment in the "plastic core" zone of action, as the damage accumulates, the parameter $\omega$ reaches a critical value of 1 .

As a result, a discrete fracture of the material occurs on a segment of $r_{\mathrm{c}}$ length, and the initial crack is incremented by this length. After that, a "plastic core" of $r_{\mathrm{c}}$ length different from the previous one is formed in the newly formed crack tip, additional damage is accumulated and the crack development process continues until the stress level in the nett 
section weakened by the crack reaches the critical value. The time accumulated by this moment is calculated by the formula (20) and characterizes the crack growth time from the initial crack-like defect to its critical length.

\section{References}

1. R.V. Gol'dshtejn, M.N. Perel'muter, Continuous Computational Mechanics, 2, 22-39 (2009)

2. R. Goldstein, E. Shifrin, P. Shushpannikov, Comptes Rendus Acad. Sciences. Paris, 336(1), 108-117 (2008)

3. F.D. Corso, D. Bigoni, J. Mech. Phys.Solids, 56, 815-838 (2008)

4. H.B.H. Hamouda, L. Laiarinandrasana, R. Piqiues, Int. J. of Pressure Vessels and Piping, 86(2), 228-238 (2009).

5. M. Rahman, J.W. Hancock, Int. J. Solids and Structures, 43, 3692-3704 (2006)

6. H. Liu, B. Rui, J. Zhang, B. Fei, International Journal of Fatigue, 59, 34-42 (2014)

7. S.Ju. Googe, I.S. Tabolin, E.I. Shirjaev, L.B. Shron, Bulletin of the Kuzbass State Technical University 1, 137-140 (2012)

8. Parton V.Z., Morozov E.A. Mehanika uprugoplasticheskogo razrushenija (M.: LKI, (2008)

9. G. R. Irwin, In Proceeding of the Seventh Sagamore Ordnance Materials Conference, 4, 63-78 (1960)

10. J.W. Hutchinson, J. Mech. Phys. Solids, 16, 13-31 (1968)

11. J. R. Rice, G.F. Rosengren, J. Mech. Phys. Solids, 16, 1-12 (1968)

12. L.V. Stepanova, Proceedings CanCNSM Vancouver, 2, 523-532 (2002)

13. K.A. Agahi, Yy.G. Basalov, V.N. Kuznecov, L.V. Fomin, Bulletin of the Samara State Technical University, 2(19), 243-247 (2009)

14. L.V. Stepanova, S.A. Igonin, Bulletin of the Samara State Technical University, 3(114), 97-114 (2014)

15. A. Saxena, Int J Fract, 191, 31-51 (2015)

16. V. Kalyanasundaram, A. Saxena, S. Narasimhachary, B. Dogan, ASTM STP, $153923-$ 40 (2011)

17. H. Riedel, Encyclopedia of Materials: Science and Technology, 1767-1773 (2008)

18. J. Pan, P.C. Lin, Engng. Fracture Mechanics, 73, 1797-1813 (2006)

19. G.C. Sih, Theoretical and Applied Fracture Mechanics, 51, 11-32 (2009)

20. H.Y. Subramanya, S. Viswanatan, R. Narasimhan, Intern. J. of Fracture, 136, 167-185 (2005) 